\title{
Gender Detection using Machine Learning Techniques and Delaunay Triangulation
}

\author{
Sarthak Gupta \\ Guru TeghBahadur Institute of Technology, \\ GGSIPU, New- Delhi, India
}

\begin{abstract}
Data mining today is being used widely in diverse areas. For example: fraudulent systems, recommender systems, disease prediction, and numerous other applications. One such application is exploited in this article. This paper presents an approach to detect gender of a person through frontal facial image, using techniques of data mining and Delaunay triangulation. Gender prediction can prove to be a very useful technique in HCI (Human Computer Interaction) Systems. Classification, being a very power technique in data mining to group categorical data, is used here to classify a gender as either male, or female. Various classification algorithms such as Functional Trees, AdaBoost, J48, and few others are used to gauge the maximum accuracy. The model used in this paper is robust and attains accuracy level of $\mathbf{9 3 . 8 2 8 3 \%}$ along with relative scale invariance. Details of the prediction model and results are reported herein.
\end{abstract}

\section{General Terms}

Data mining, classification algorithms, WEKA tool, gender detection, Machine learning

\section{Keywords}

Functional Trees, J48, Naïve Bayes, Machine Learning, Gender Classification, WEKA, Machine learning

\section{INTRODUCTION}

Gender Classification has become area of extensive research due to it's increasingly powerful applications. Automated gender classification has attracted much attention over the last ten years since augmenting this ability with applications specific to a particular gender can provide a more userfriendly environment and human-like interaction [1], [2].

Till date, the work has been emphasized on gender recognition through visual observation, but now, it has to be emphasized to computer, to perform this task.It is observable that our behavior and social interaction are greatly influenced by genders of people whom we intend to interact with. Hence a successful gender recognition system could have great impact in improving human computer interaction systems in such a way as to make them be more user-friendly and acting more human-like. Over the past decades, there have been significant advances in facial image processing, especially, in a face detection area where a number of fast and robust algorithms [3], [4] have been proposed for practical applications [5]. As a result, a number of research areas attempting to extend the works have been emerging, face recognition, facial expression recognition and gender recognition, for example.

Conforming to the practicality of gender prediction, it is imperative to improve the algorithms from time to time in order to achieve higher accuracy levels and build more robust and accurate systems.
Based on the types of features used, facial feature extraction approaches can be roughly divided into two different categories: geometric feature-based methods and appearancebased methods [6], [7]. Geometric features refer to distance between various facial features such as eyes, nose, chin and lips. Facial features can be extracted from facial image using Viola Jones algorithm that returns the coordinates of various features. Euclidean distance then can be calculated between the detected features, along with Delaunay triangulation calculation. For the sake of accuracy, it has to be taken care of that facial features are detected accurately, and hence, every image from database was manually picked and mined, adhering to proper detection. Few adjustments had to me made in algorithm for some specific images, but every image was manually scrutinized and mined carefully.

In a Delaunay triangulation of facial features for a face, the Delaunay triangles have different sizes in different areas. By classifying the size of the Delaunay triangles, the different facial figures are separated into a number of regions [8]. In a well-separated point set, Delaunay triangles can beclassified into two types. A foreground triangle (F-T) is a Delaunay triangle in a cluster [8]abackground triangle (BT)is a Delaunay triangle outside any cluster. Highly densepoints form F-Ts, while sparse points constitute B-Ts. Clear boundaries can be found to differentiate the two typesof triangles.[8].

Machine Learning is a field of computer science that evolved from field of pattern recognition and computational learning theory in Artificial Intelligence Machine Learning explores the study of algorithms that can learn and make predictions from available raw data. This article has exploited the capabilities of machine learning. Various Machine learning algorithms are deployed for pattern recognition from the data mined from facial images. AdaBoost or adaptive boosting and other algorithms including functional trees are used to measure maximum accuracy levels.

FEI face database [27] database has been used that contains 200 frontal facial images consisting of 100 male faces and 100 female faces. Each subject has a normal expression and a smiling expression associated. The images were preprocessed using MATLAB in order to assure the uniform light effects. Features described in section 3.2 were extracted with the help of MATLAB and loaded into database, from where they were fetched and analyzed. Functional Trees were used to classify the detected gender as either male or female. Highest accuracy of $\mathbf{9 3 . 8 2 8 3 \%}$ is achieved in comparison to J48, Random Forest and others summarized in section 4. WEKA (Waikato Environment for Knowledge Analysis) is used as the tool for analysis. The accuracy level is improved in comparison to previous researched performed by [14][24]

Following is the organization of rest of the paper:- 
Section 2 describes about literature Survey; the researches that have been performed in this area. Section 3 elucidates the methodology and model proposed in this research. Section 4 and 5 summarizes the results of the research. Section 6 throws light on any future work scope that can augment this research. Concluding the paper with Section 7, it acknowledges the work of various researches that have been conducive in concocting this article.

\section{LITERATURE SURVEY}

There have been several efforts towards detecting the gender through facial image. For example, the gender classification based on human faces has been extensively researched in the article $[9,10]$. Theorypresented two popular methods. The former is proposedby Moghaddam et al. [10] where a Support Vector Machine(SVM) is utilized for gender classification based on thumbnailface images. The latter was presented by Baluja et al.[9] who applied the AdaBoost algorithm for gender prediction.

Gao et al. [11] performed face-based gender classificationon consumer images acquired from a multi-ethnic facedatabase. To overcome the non-uniformity of pose, expression, and illumination changes, they proposed the usage ofActive Shape Models (ASM) to normalize facial texture.The work concluded that the consideration of ethnic factorscan help improve gender classification accuracy in a multiethnicenvironment.

Zehang Sun et al, [12] proposed Gender classification from frontal face images using genetic feature subset selection. They used Principal Component Analysis (PCA) to represent each individual image as a feature vector in a low dimensional space and based on Genetic algorithms, a subset of features are extracted from the low-dimensional representation by ignoring certain eigenvectors which are not needed for classifying facial feature information.

Multi-view gender classification by focusing on shape and texture data for representing facial images was proposed by Hui-Ching Lain and Buo-Liang Lu [13]. In this, the facial area is divided into small regions by using local binary pattern (LBP) histograms.

Md. Hafizur Rahman et al, [14] performed Face Detection and Sex Identification from Color Images using AdaBoost with SVM based Component Classifier and achieved a maximum accuracy level of $89.51 \%$ for male prediction and $87.80 \%$ for female prediction.

In previous work $[15,16]$ geometry featureswere used as a priori knowledge to help improve classificationperformance, none of the aforementioned approaches,unlike this work, focused explicitly and solely on facialmetrology as a means for gender classification. Perhaps this research is more closely related to earlier research by Yi Xiao1et al. [8] on facial Feature Location with Delaunay Triangulation/Voronoi Diagram Calculation, where theylocate "key points" of facial features using Delaunay Triangulation and Voronoi Diagram technique. However, this article takes a more comprehensive lookat the explicit use of facial geometry in solving the problem.

\section{METHODOLOGY}

As described in section 1, this article exploits the FEI database [27] that consists of 100 female and 100 male, colored frontal facial images, with each subject having two expressions associated. The geometric feature-based method has been deployed that calculates the Euclidian distance between the detected facial features. The following flowchart in figure 1describes about the general outline:-

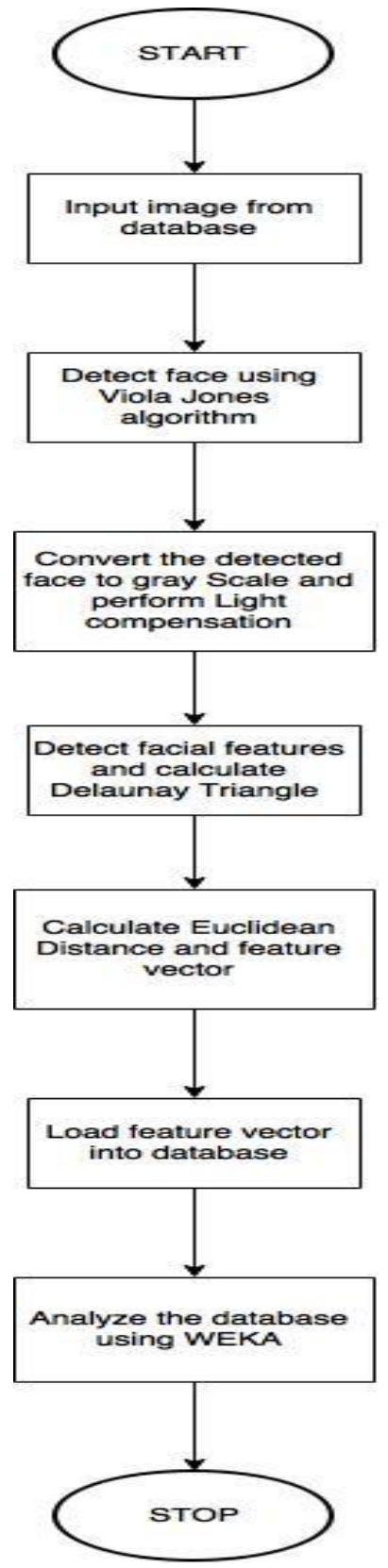

Figure: 1

Flow chart describing flow of model

The above phases are described in following sub-sections.

\subsection{Light Compensation}

All too often, pictures takes from digital cameras produce undesirable light effects, contributing to light and dark bands in some images. Hence, the need arises for Light Compensation in order to detect features correctly. The problem of elimination of non-standard illumination is one of the most complicated problems in the area of computer vision. Due to the complex illuminated environment in the real world. According to $[17,18,19,20$ and 21], the lighting compensation (LC) algorithm is very efficient in enhancing and restoring the natural colors into the images which are taken in darker and varying lighting conditions. The 
implemented LC algorithm is based on the assumption that the spatial average of surface reflectance in a scene is achromatic. Since the light reflected from an achromatic surface is changed equally at all wavelengths, it follows that the spatial average of the light leaving the scene will be the color of the incident illumination [22]. The Light Compensation algorithm can be defined as described below:

$$
\begin{aligned}
& S_{C}=\frac{C_{\text {std }}}{C_{\text {avg }}} \\
& C_{\text {avg }}=\frac{\sum_{i=1}^{i=m}\left(C_{i}\right) C_{i}>0}{\sum_{i=1}^{i=m}(1) C_{i}>0} \\
& C_{\text {std }}=\frac{\sum_{i=1}^{i=m}\left[\max \left(R_{i}, G_{i}, B_{i}\right)+\min \left(R_{i}, G_{i}, B_{i}\right)\right]}{2 \times n} \\
& n=m-\sum_{i=1}^{i=m}(1) R_{i}=G_{i}=B_{i}=0
\end{aligned}
$$

Where, $S_{\mathrm{c}}$ stands for the scale factor for one specific channel of $\mathrm{R}, \mathrm{G}$ or B. The $\mathrm{C}_{\text {stdand }} \mathrm{C}_{\text {avgseparately stand for the standard }}$ mean gray value of the specific channel and the mean value non-black pixels in the same channel[22]. The $m$ stands for the number of pixels in the image, $\mathrm{n}$ stands for the number of non-black pixels in the image [22]. This is to solve the over compensation problem for images with dark background, i.e., images taken at night [22]. By calculating the average of the maximum and minimum channel percentage, an adaptive mean gray value of the whole image is gained. [22]

\subsection{Face Detection and Feature Extraction}

For detection of facial features, it is first necessary to detect face from the input image. Therefore, Viola Jones Algorithm is deployed to detect face as well as facial features from the input image. It is a robust algorithm for detecting faces, proposed by Paul Viola and Michael J. Jones. Face detection is shown in figure 2a.The detected facial image was then, converted into Grayscale counterpart, for better performance.

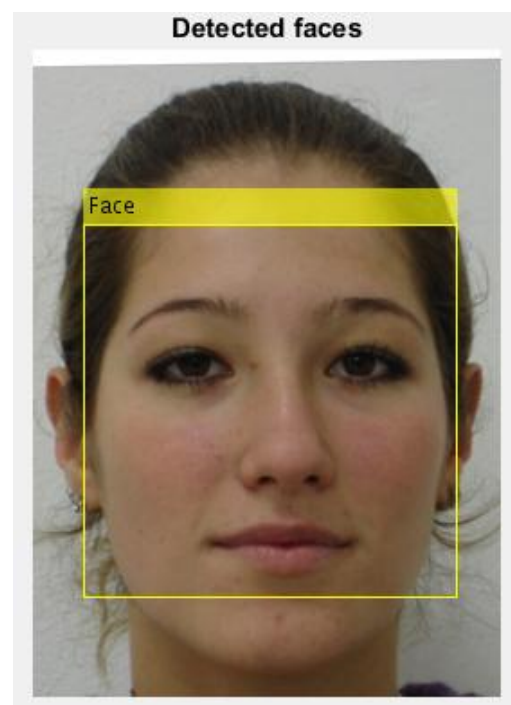

Figure: $2 a$

Face detected from input image using Viola-Jones Algorithm
Here is the list of features extracted from facial image for analysis:-

Table: 1

Features vector extracted from facial image

\begin{tabular}{|l|l|l|}
\hline S.NO & NAME & DESCRIPTION \\
\hline 1 & EE & $\begin{array}{l}\text { Euclidean distance between } \\
\text { eyes }\end{array}$ \\
\hline 2 & LEFC & $\begin{array}{l}\text { Euclidean distance between } \\
\text { Left-eye and face center }\end{array}$ \\
\hline 4 & LENC & $\begin{array}{l}\text { Euclidean distance between } \\
\text { Right-eye and face center } \\
\text { Left-eye and Nose center }\end{array}$ \\
\hline 5 & RENC & $\begin{array}{l}\text { Euclidean distance between } \\
\text { Right-eye and Nose center }\end{array}$ \\
\hline 6 & LEMC & $\begin{array}{l}\text { Euclidean distance between } \\
\text { Left-eye and center of Lips }\end{array}$ \\
\hline 7 & REMC & $\begin{array}{l}\text { Euclidean distance between } \\
\text { right-eye and center of Lips }\end{array}$ \\
\hline 9 & NCMC & $\begin{array}{l}\text { Euclidean distance between } \\
\text { center of Nose and Lips }\end{array}$ \\
\hline
\end{tabular}

Euclidean distance is defined as: -

$$
\begin{aligned}
& D(p, q)=D(q, p)= \\
& \sqrt{\left(q_{1}-p_{1}\right)^{2}+\left(q_{2}-p_{2}\right)^{2}+\ldots+\left(q_{n}-p_{n}\right)^{2}}
\end{aligned}
$$

Where, In Cartesian Coordinates, $p=\left(p_{1}, p_{2}, p_{3}, \ldots, p_{n}\right)$ and $q=\left(q_{1}, q_{2}, q_{3}, \ldots, q_{n}\right)$ are two points in Euclidean nspace, $\quad D(p, q)=D(q, p)=$ Euclidean distance between points $\mathrm{p}$ and $\mathrm{q}$.

\subsection{Delaunay Triangulation}

In a point set $P$, its Delaunay Triangulation is defined as[8]:-

$$
D T=\left\{\begin{array}{l}
T\left(p_{i}, p_{j}, p_{k}\right) \mid p_{i} \in P, p_{j} \in P, p_{k} \in P, \\
C\left(p_{i}, p_{j}, p_{k}\right) \cap P \backslash\left(p_{i}, p_{j}, p_{k}\right)=\phi
\end{array}\right\}
$$

Where, $C\left(p_{i}, p_{j}, p_{k}\right)$ is the circle circumscribed by thethree vertices $p_{i}, p_{j}, p_{k}$, which form a Delaunay triangle $T\left(\mathrm{p}_{\mathrm{i}}, p_{j}, p_{k}\right)$. A Delaunay triangulation is a triangulation $\mathrm{DT}(\mathbf{P})$ such that no point in $\mathbf{P}$ is inside the circum-hypersphere of any simplex in DT(P). It is known that there exists a unique Delaunay triangulation for $\mathbf{P}$ if $\mathbf{P}$ is a set of points in general position; that is, the affine hull of $\mathbf{P}$ is $d$-dimensional and no set of $d+2$ points in Plie on the boundary of a ball whose interior does not intersect $\mathbf{P}$. Delaunay Triangulation is then calculated for the Euclidean distances calculated before. The image $2 b[23]$ shows Delaunay Triangulation. 


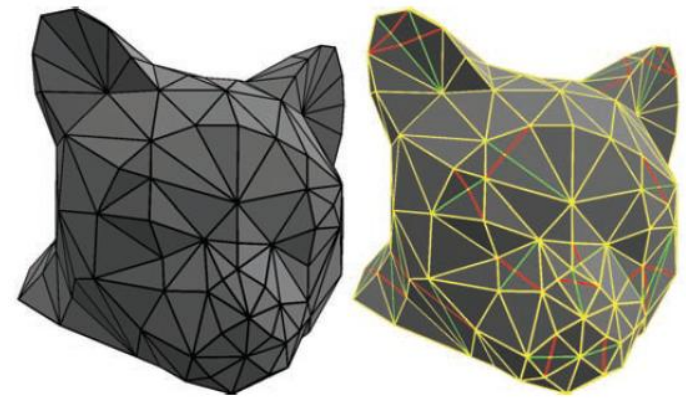

Figure 1: Left: carrier of the (cat head) surface as defined by the original embedded mesh. Right: the intrinsic Delaunay triangula${ }^{\text {tion }} \mathbf{2 b}$ edges are part of the original and intrinsic Delaunay triangumuon; red edges resulted from flipping; and green edges denote original edges which are not part of the intrinsic Delaunay triangulation. Note that the red edges are geodesic lines on the original surface.

One of the key properties of Delaunay triangulations is the empty circumscribing sphere condition. In $\mathrm{R} \sim$ there is a unique sphere with any given $\mathrm{n}+1$ points on its boundary. Delaunay Triangulation method has shown remarkably exceptional results. The distances calculated are then loaded into the database, from where they were fetched and analyzed. Delaunay Triangles calculated for input image has been shown in figure $2 \mathrm{c}$ (male) and figure $2 \mathrm{~d}$ (female). As seen in figure, features detected from face have been labeled in yellow boxes. 1 refers to left eye, 2 refer to right eye, 3 refer to nose and 4 refer to lips.

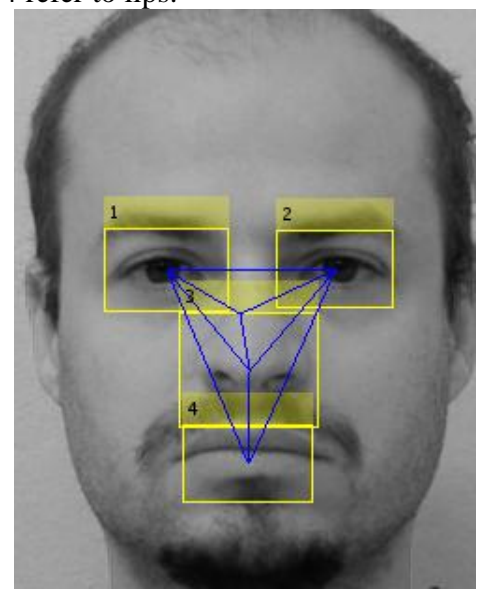

Figure 2c
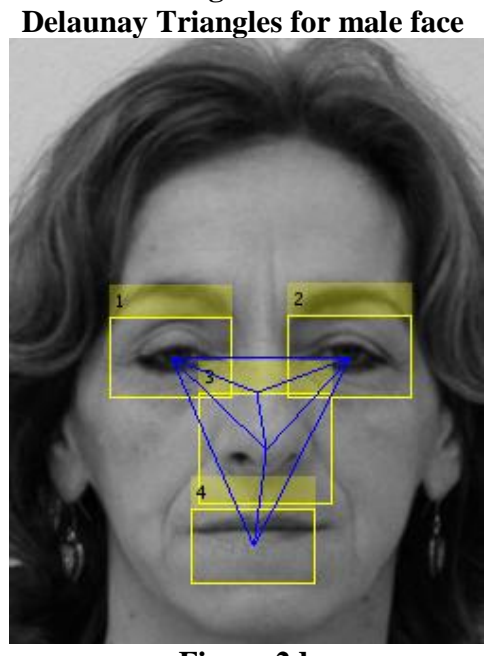

Figure 2d

Delaunay Triangles for Female face

\subsection{Analysis and Classification}

After extracting all the relevant features, Machine Learning algorithms were applied, that classifies the image as a Male or a Female face. The WEKA tool was used to apply various algorithms. Functional Trees algorithm outperformed the second best by $\mathbf{8 . 0 8 4 8} \%$, and showed highest accuracy level. The accuracy obtained in this research is more than that of $[14,24]$. Several other algorithms were also deployed for the comparison purposes. These include, Random forest, Naïve Bayes, AdaBoost and J48. The results are formally represented in $4^{\text {th }}$ section of this article.

\subsubsection{Functional Trees}

In the context of machine learning, it is imperative to first classify the given problem as a regression problem, or a classification problem. If the target class variable takes a value ' $k$ ', where $\mathrm{k} \in \mathrm{S}\{0,1, \ldots \mathrm{N}\}$ and $\mathrm{S}$ is a set consisting of finite number of values ' $\mathrm{N}$ ', then, given problem is a classification problem. If the target class variable takes any continuous value, then the given problem is termed as "Regression" problem.Given a set of examples and an attribute constructor, the general algorithm [25] used to build a functional tree is described as:-

\section{Function GrowTree(Dataset, Constructor)}

1. If Stop_Criterion(Dataset)

Return a Leaf Node with a Constant Value

2. Construct a model $\phi$ using Constructor

3. For each example $\mathrm{x} \in$ DataSet

$$
\begin{aligned}
& \text { - } \quad \text { Compute } \hat{y}=\phi(x) \\
& \text { - } \quad \text { Extend } \mathrm{x} \text { with new attributes } \hat{y}
\end{aligned}
$$

4. Select the attribute of original as well as of newly constructed attributes that maximizes some meritfunction.

5. For each partition iof the DataSet using the selected attribute

$$
\begin{aligned}
& \text { Tree }_{\mathrm{i}}=\text { GrowTree}_{\left(\text {DataSet }_{\mathrm{i}}\right.}, \\
& \text { Constructor })
\end{aligned}
$$

6. Return a Tree, as a decision node based on the selected attribute, containing the $\phi$ model, and descendants Tree ${ }_{i}$

\section{End Function}

\subsubsection{AdaBoost}

The AdaBoost algorithm proposed by Yoav Freund and Robert Schapire is one of the most important ensemble methods, since it has solid theoretical foundation, very accurate prediction, great simplicity (Schapire said it needs only "just 10 lines of code"), and wide and successful applications [26]. XindongWu et al. in paper [26] has described AdaBoost algorithm as follows:-

Let $X$ denote the instance space and $Y$ the set of class labels. Assume $Y=\{-1,+1\}$. Given a weak or base learning algorithm and a training set $\left\{\left(x_{1}, y_{1}\right),\left(x_{2}, y_{2}\right) \ldots,\left(x_{m}, y_{m}\right)\right\}$ where $x_{i} \in X$ and $y_{i} \in Y(i=1, \ldots, m)$, the AdaBoost algorithm works as follows. First, it assigns equal weights to all the training examples $\left(\boldsymbol{x}_{i}, y_{i}\right)(i \in\{1, \ldots, m\})$. Denote the distribution of the weights at the $t$-th learning round as $D t$. From the training set and $D t$ the algorithm generates a weak or 
base learner $h t: X \rightarrow Y$ by calling the base learning algorithm. Then, it uses the training examples to test $h t$, and the weights of the incorrectly classified examples will be increased. Thus, an updated weight distribution $D t+1$ is obtained. From the training set and $D t+1$ AdaBoost generates another weak learner by calling the base learning algorithm again. Such a process is repeated for $T$ rounds, and the final model is derived by weighted majority voting of the $T$ weak learners, where the weights of the learners are determined during the training process. The pseudo code for the algorithm is presented in [26] as:-

Input: $\quad$ Data set $\mathrm{D}=\left\{\left(x_{1}, y_{1}\right),\left(x_{2}, y_{2}\right), \ldots,\left(x_{m}, y_{m}\right)\right\}$;

Base learning algorithm $\boldsymbol{L}$

Number of learning rounds $\boldsymbol{T}$.

\section{Process:}

$D_{1}(i)=1 / m \quad \%$ Initialize the weight Distribution

For $\mathrm{t}=1, \ldots, \mathrm{T}$ :

$h_{t}=L\left(D, D_{t}\right) ; \%$ Train a weak learner $h_{t}$ from D usingdistributón $\mathrm{D}_{\mathrm{t}}$

$\varepsilon_{t}=\operatorname{Pr}_{i \sim D_{i}}\left[h_{t}\left(x_{i} \neq y_{i}\right)\right] ; \%$ Measurethe error of $h_{t}$

$\alpha_{t}=\frac{1}{2} \ln \left(\frac{1-\varepsilon_{t}}{\varepsilon_{t}}\right) ; \%$ Determinethe weightof $h_{t}$

$D_{t+1}(i)=\frac{D_{t(i)}}{Z_{t}} \times\left\{\begin{array}{ll}\exp \left(-\alpha_{t}\right) & \text { if } h_{t}\left(x_{i}\right)=y_{i} \\ \exp \left(\alpha_{t}\right) & \text { if } h_{t}\left(x_{i}\right) \neq y_{i}\end{array}\right\}$

$D_{t+1}(i)=\frac{D_{t}(i) \exp \left(-\alpha_{t} y_{i} h_{t}\left(x_{i}\right)\right)}{Z_{t}}$

End

Output: $H(x)=\operatorname{sign}\left(\sum_{t=1}^{T} \alpha_{t} h_{t}(x)\right)$

\section{RESULTS}

Several Classification algorithms were used to monitor the accuracy levels. The results are summarized in table 2 .

Table: 2

Summarized results

\begin{tabular}{|l|l|l|}
\hline S.no. & Classifier & Accuracy \\
\hline 1 & Functional Trees & $\mathbf{9 3 . 8 2 8 3 \%}$ \\
\hline 2 & Random Forest & $\mathbf{8 5 . 7 4 7 5 \%}$ \\
\hline 3 & Naïve Bayes & $\mathbf{8 4 . 7 4 7 5 \%}$ \\
\hline 4 & AdaBoost & $\mathbf{8 3 . 7 3 7 4 \%}$ \\
\hline 5 & J48 & $\mathbf{8 0 . 6 9 7 0 \%}$ \\
\hline
\end{tabular}

Figure 3 provides a performance comparison of various algorithms

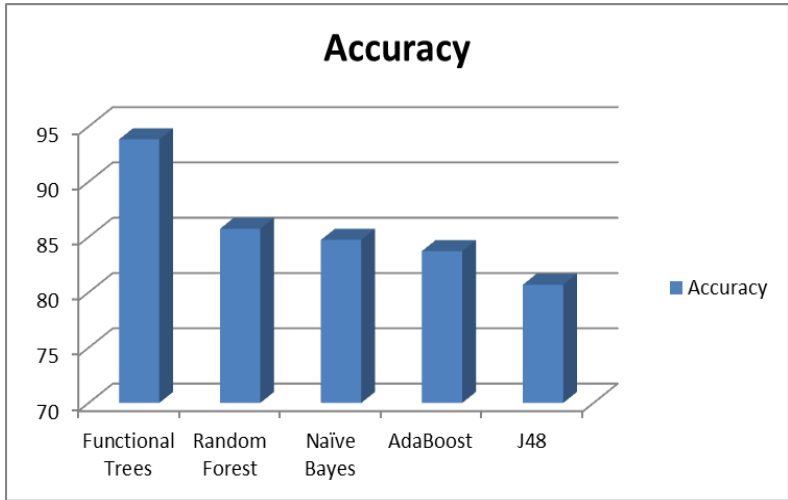

Figure 3

Performance Comparison

\section{CONCLUSION}

The results show that Delaunay Triangulation indeed can be used for gender prediction. From the table 2, it can be seen that Functional Trees algorithm performed best, and accuracy of $\mathbf{9 3 . 8 2 8 3 \%}$ is observed, which shows improvement in results as compared to [14] and [24]. Gender prediction finds importance in diverse areas such as HCI, AI, and other related fields, hence; there is a need to constantly upgrade existing methods to a new level of perfection and higher accuracy levels.

\section{FUTURE WORK}

The possibility of gender detection through Euclidean distance and Delaunay Triangulation has been demonstrated in this article. The accuracy levels are satisfactory, but the question arises: Whether the accuracy can be improved any further? The next steps in the research would be to address the solution to better accuracy levels with improved algorithms. Another initiative would be to detect the age of the subject through the same frontal facial image, as used in gender detection. Age prediction can prove to be a very useful technique in order to improve the HCI interaction and make it more realistic. Age prediction can also be exploited in surveillance systems where there prevails the need to detect the subject's age. Machine Learning has the capability to address most of the real world problems by virtue of robust algorithms and thus, age prediction can also be solved through the techniques of Machine learning, which will be addressed in the next phases of this research.

\section{REFERENCES}

[1] H. C. Kim, D. Kim, Z. Ghahramani, S. Y. Bang, "Appearance-based gender classification with gaussian processes," Pattern Recognition Letters, Vol. 27, No. 6, pp. 618-626, 2006.

[2] F. Ahmed, M. H. Kabir, "Facial feature representation with directional ternary pattern (dtp): Application to gender classification," in Proceedings of the IEEE International Conference on Information Reuse and Integration, USA, 2012, pp. 159-164.

[3] Viola, P. \& Jones, M., 2001. Rapid object detection using a boosted cascade of simple features. Proceedings of the 2001 IEEE Computer Society Conference on Computer Vision and Pattern Recognition, 2001. CVPR 2001., 1, pp.511-518.

[4] Huang, C. et al., 2004. Boosting nested cascade detector for multi-view face detection. Proceedings of the 17th International Conference on Pattern Recognition, 2004. ICPR 2004., 2, pp.415-418. 
[5] Yang, Z., Ming, L. \&Haizhou, A., 2006. An Experimental Study on Automatic Face Gender Classification. 18th International Conference on Pattern Recognition, 2006. ICPR 2006., pp.1099-1102.

[6] H. C. Kim, D. Kim, Z. Ghahramani, S. Y. Bang, "Appearance-based gender classification with Gaussian processes," Pattern Recognition Letters, Vol. 27, No. 6, pp. 618-626, 2006.

[7] F. Ahmed, H. Bari, E. Hossain, "Person-independent facial expression recognition based on compound local binary pattern (clbp)," International Arab Journal of Information Technology, Vol. 11, No. 2, 2013.

[8] Yi Xiao, Hong Yan, 2002, Facial Feature Location with Delaunay Triangulation/Voronoi Diagram Calculation, School of Electrical \& Information Engineering, University of Sydney, NSW 2006, Australia

[9] S. Baluja and H. A. Rowley. Boosting sex identification performance. IJCV, 71(1):111-119, 2007.

[10] B. Moghaddam and M.-H. Yang. Learning gender with support faces. IEEE TPAMI, 24:707-711, 2002.

[11] W. Gao and H. Ai. Face gender classification on consumer images in a multiethnic environment. In ICB, pages $169-178,2009$.

[12] RoytatsuIga, Kyoko Izumi, Hisanori Hayashi, GentaroFukano and TestsuyaOhtani, "Gender and Age Estimation from Face Images", International Conference on The Society of Instrument and Control Engineering, pp. 756-761, August, 2003.

[13] Hui-Cheng Lain and Bao-Liang Lu, "Multi-View Gender Classification using Local Binary Patterns and Support Vector Machines", International Conference on Neural Networks, pp. 202-209, 2006.

[14] Md. Hafizur Rahman et al., 2013, "Face Detection and Sex Identification from Color Images using AdaBoost with SVM based Component Classifier", International Journal of Computer Applications (0975 - 8887), Volume 76- No.3, August 2013

[15] Y. Saatci and C. Town. Cascaded classification of gender and facial expression using active appearance models. In FGR, 2006

[16] Z. Xu, L. Lu, and P. Shi. A hybrid approach for gender classification from face images. In ICPR, 2008.
[17] Gundimada, S. and Asari, V., (2004), Face Detection Technique Based on Rotation Invariant Wavelet Features, Proceedings of ITCC International Conference on Information Technology: Coding and Computing, pp: $157-158$

[18] Hsu, R. L., Abdel-Mottaleb, M. and Jain, A. K. (2002), Face Detection in Color Images, IEEE Transactions on Pattern Analysis and Machine Intelligence, Vol. 24, No. 5, pp: 696-706.

[19] Xie, X. and Kin-Man, L. (2004), An Efficient Illumination Compensation Scheme for Face Recognition. 8th Control, Automation, Robotics and Vision Conference (ICARCV 2004), pp: 1240 - 1243

[20] Pai, Y., Ruan, S., Shie, M. and Liu, Y. (2006), A Simple and Accurate Color Face Detection Algorithm in Complex Background, IEEE International Conference on Multimedia and Expo. Toronto, ON, Canada, pp: 15451548 .

[21] Gundimada, S. Li Tao and Asari, V. (2004), Face Detection Technique Based on Intensity and Skin Color Distribution, ICIP '04. International Conference on Image Processing, Vol.2, pp: 1413 - 1416

[22] Ahmed M. Mharib et al., 2011, The Impact of Light Compensation on the Performance of Parametric Skin Detection Model, International Journal of Signal Processing, Image Processing and Pattern RecognitionVol. 4, No. 4, December, 2011

[23] Matthew Fisher et al., 2006, An Algorithm for the Construction of Intrinsic Delaunay Triangulations with Applications to Digital Geometry Processing, SIGGRAPH 2006

[24] SwathiKalam et al. Gender Classification using Geometric Facial Features, International Journal of Computer Applications (0975 - 8887), Volume 85 - No 7, January 2014

[25] JOA O GAMA, 2004, Functional Trees, University of Porto, Rua Campo Alegre 823, 4150 Porto, Portugal, Machine Learning, 55, 219-250, 2004

[26] XindongWu et al., 2008, Top 10 algorithms in data mining, Springer, KnowlInfSyst (2008) 14:1-37

[27] FEI face database, http://fei.edu.br/ cet/facedatabase.html 